\title{
A MOTZKIN-TYPE THEOREM FOR CLOSED NONCONVEX SETS
}

\author{
L. CALABI AND W. E. HARTNETT ${ }^{1}$
}

Introduction. Bouligand [1] recognized the importance of the nearest-points mapping for a closed set $X$ and the set $S_{X}$ of points with more than one nearest point in $X$ for the study of geometry. Later Motzkin [3], [4] used them in the proof of his theorem characterizing closed convex sets. We use them to show, essentially, that $S_{X}$ characterizes the complement of $X$ in its convex hull. Our result includes the Motzkin theorem as a special case and yields a theorem of Valentine [5] as a corollary. The original motivation and background for our work can be found in [2].

The statement of the theorem. To every closed set $A$ of the Euclidean $n$-dimensional space $E$ we associate its closed convex hull $C(A)$ and its convex deficiency $D=D(A)=C(A) \backslash A$. We denote by $\pi$ the nearest-points mapping $A$ and by $r$ the distance from $A$ :

$$
r(x)=d(x, A), \quad \pi(x)=\{y: y \in A, d(x, y)=r(x)\},
$$

where $d$ denotes the Euclidean distance.

We let $B(x)$ denote the closed ball around $x$ of radius $r(x)$ and $B^{0}(x)$ denote its interior. Observe that $B^{0}(x) \cap A=\varnothing$ and $B(x) \cap A$ $=\pi x$. We shall say that $x \notin A$ is a skeletal point of $A$ iff $B(x)$ is contained in no other $B\left(x^{\prime}\right)$. The set of all skeletal points of $A$ is the skeleton of $A$ and is denoted by $S$. The skeletal pair of $A$ is $(S, q)$, where $q$ is the restriction of $r$ to $S$. Clearly $S$ contains all points having more than one nearest point in $A$; in fact, as already shown by Motzkin [3], such points form a dense subset of $S$.

Our main result may now be stated.

THEOREM. Two closed subsets of $E$ have the same convex deficiency if and only if they have the same skeletal pair.

The proof of the theorem follows.

$D$ determines $(S, q)$. If $x, y \in E$ and $x \neq y$, we let $[x, y]$ denote the segment with endpoints $x$ and $y$ and set $[x, y)=[x, y] \backslash\{y\}$ and $(x, y]=[x, y] \backslash\{x\}$. We let $[y, x\rangle$ denote the closed ray with end point

Presented to the Society, March 31, 1966 under the title Extension to nonconvex, closed sets of Motzkin theorem on convexity; received by the editors August 26, 1967.

${ }^{1}$ Research supported by the Air Force Cambridge Research Laboratories. 
$y$ and set $(y, x\rangle=[y, x\rangle \backslash\{y\}$. For $y \in A, \pi^{-}(y)=\{x: x \in E, y \in \pi x\}$. For each set $X$ we put $X^{*}=\{x: x \in E, d(x, C(X))=d(x, y)$ with $y \in X\}$. Notice that $X=C(X) \cap X^{*}$.

Lemma 1. If $D$ is the convex deficiency of $A$, we have:

(a) $D^{*}$ is the complement of $A^{*}$.

(b) $A^{*}=A \cup\{x: x \in E$, if $y \neq x$ and $y \in \pi x$, then $\pi x=\{y\}$ and $\left.[y, x\rangle \subset \pi^{-}(y)\right\}$.

(c) $D^{*}=\left\{x: x \notin A\right.$, if $y \in \pi x$, then $\pi^{-}(y) \cap[y, x\rangle=[y, z]$ for some $\left.z\right\}$.

Proof. Observe that $x \in A^{*}$ iff $d(x, C(A))=d(x, A)$. Hence, because $d(x, C(A)) \leq d(x, A), A^{*}=E \backslash D^{*}$ iff $d(x, C(A))<d(x, A)$ for each $x \in D^{*}$.

If $d(x, C(A))<d(x, A)$, then $d(x, C(A))=d(x, y)$ for some $y \in C(A) \backslash A=D$. Thus $d(x, y) \leq d(x, C(A)) \leq d(x, C(D)) \leq d(x, y)$, since also $y \in C(D)$. Consequently $x \in D^{*}$.

Conversely, to prove that $x \in D^{*}$ implies $d(x, C(A))<d(x, A)$, we prove that $x \in D^{*}$ implies $d(x, C(D))=d(x, C(A))=d(x, y)$ for some $y \in D$. If $x \in D$, that statement is trivial. Assume then $x \in D^{*} \backslash D$, and hence also $x \in C(D)$. Then, for some $y \in D, d(x, C(D))=d(x, y)$. Let $H_{y}$ be the hyperplane of support for $C(D)$ at $y$ orthogonal to $[y, z]$ and let $E_{y}$ be the closed half space bounded by $H_{y}$ and containing $C(D)$. If $y^{\prime} \in A \backslash E_{y}$, then $\left[y, y^{\prime}\right] \subset C(A)$ and, since $D \subset E_{y}$, $\left(y, y^{\prime}\right] \subset A$. But $A$ is closed, and hence $y \in A$, contradicting the fact that $y \in D$. Then $A \subset E_{y}, C(A) \subset E_{y}$ and $d(x, C(D))=d(x, C(A))$ $=d(x, y)$ with $y \in D$. If $d(x, C(A))=d(x, y)=d(x, A)$, then $y \in A$ because $A$ is closed. Hence $d(x, C(A))<d(x, A)$ and (a) is established.

To prove (b) it is enough to show that $A^{*}$ contains the second set at the right of the equal sign so we pick $x$ in the set. Then the hyperplane orthogonal to $[y, x\rangle$ passing through $y$ is a hyperplane of support for $A$ and hence for $C(A)$. Thus $y \in C(A)$ and $d(x, A)=d(x, C(A))$, that is $x \in A^{*}$. Statement (c) follows at once from (a) and (b).

We set $F(D)=($ bd $D) \backslash D$ and observe that $D=\varnothing$ iff $D^{*}=\varnothing$ iff $F(D)=\varnothing$.

Lemia 2. If $A$ has convex deficiency $D$, then for $x \in D^{*}$ we have $r(x)$ $=d(x, F(D))$.

Proof. Suppose $y \in \pi x$. If $x \in D \subset C(A)$, then $(y, x] \subset D, y \notin D$, and hence $y \in F(D)$. If $x \in D^{*} \backslash D$, let $y^{\prime}=\pi_{0} x$ be the projection of $x$ into $C(D) \subset C(A)$. Then $\left[y, y^{\prime}\right] \subset C(A),\left(y, y^{\prime}\right] \subset C(A) \backslash A=D, y \notin D$, and so $y \in F(D)$.

If $(S, q)$ is the skeletal pair of $A$, we let $P(A)=\cup\{(y, x]$ : $y \in \pi x, x \in S\}$. We then have the following result: 
Lemma 3. Suppose that $A$ has convex deficiency $D$ and skeletal pair $(S, q)$. Then $S \subset P(A)=D^{*}$.

Proof. The inclusion is trivial. The equality follows from Lemma 1 (c) and the observation that $x \in S$ iff $x \notin A$ and $\pi^{-}(y) \cap[y, x\rangle$ $=[y, x]$ for $y \in \pi x$.

The proof of the next lemma is immediate.

Lemma 4. The skeleton $S$ of $A$ is the set of those points $x \in D^{*}$ for which

$$
\begin{array}{rlrl}
r\left(x^{\prime}\right)+d\left(x, x^{\prime}\right) & =r(x) & & \text { if } x^{\prime} \in[y, x], \\
r(x)+d\left(x, x^{\prime}\right)>r\left(x^{\prime}\right) & & \text { if } x^{\prime} \in[y, x\rangle \backslash[y, x]
\end{array}
$$

for every $y \in \pi x$.

We can now establish the first half of the theorem. Let $A, A^{\prime}$ be two closed sets with equal convex deficiency $D$. Then $P(A)=P\left(A^{\prime}\right)$ by Lemma 3 , and $r(x)=r^{\prime}(x)$ for each $x \in P(A)$ by Lemma 2. Lemma 4 yields $S=S^{\prime}$ and consequently $q=q^{\prime}$.

$(S, q)$ determines $D$. For each set $X$ we put $B^{0}(X)=\bigcup\left\{B^{0}(x): x \in X\right\}$ and $\pi X=\bigcup\{\pi x: x \in X\}$. Notice that if $X \cap A=\varnothing$, then $B^{0}(X)$ $\cap A=\varnothing$ and $\pi X \subset$ bd $A$.

Lemma 5. Let $A$ have convex deficiency $D$ and skeletal pair $(S, q)$. Then

(a) $\pi x=B(x) \backslash B^{0}(S) \subset F(D)$ for each $x \in D^{*}$.

(b) $\mathrm{Cl} \pi S=F(D)$.

Proof. First observe that $D \subset B^{0}\left(D^{*}\right) \subset B^{0}(S)$. Because $\pi x$ $C B(x) \backslash B^{0}(S)$, it is enough to show that $B(x) \backslash B^{0}(S) \subset A$. If $x^{\prime} \in(B(x) \backslash A) \cap C(A)$, then $x^{\prime} \in D \subset B^{0}(S)$. Hence $\left(B(x) \backslash B^{0}(S)\right)$ $\cap C(A) \subset A$. If $x^{\prime} \in B(x) \backslash C(A)$, let $y \in \pi x$. Because $y \in C(A)$, $x^{\prime} \notin C(A)$, and $x \notin A^{*}$, there exists a point $y^{\prime} \in\left(y, x^{\prime}\right) \cap$ bd $C(A)$ $\subset B^{0}(x)$. Let $y_{0} \in$ bd $C(A) \cap B^{0}(x)$ be such that $d(x$, bd $C(A))$ $=d\left(x, y_{0}\right)$. Clearly, $y_{0} \in D$ because $y_{0} \in B^{0}(x)$. Let $H_{y_{0}}$ be a hyperplane of support for $C(A)$ and hence for $C(D)$ at $y_{0}$. There exists $z \notin C(D)$ such that the open ray $\left(y_{0}, z\right)$ orthogonal to $H_{y_{0}}$ at $y_{0}$ lies in $D^{*} \backslash D$. Since, for $z^{\prime} \in\left(y_{0}, z\right\rangle, d\left(z^{\prime}, y_{0}\right)=d\left(z^{\prime}, C(D)\right)<d\left(z^{\prime}, A\right)$ it is easy to see that $x^{\prime} \in B^{0}\left(z^{\prime}\right)$ for some $z^{\prime} \in\left(y_{0}, z\right\rangle$. But $B^{0}\left(z^{\prime}\right) \subset B^{0}(S)$ and hence $\left(B(x) \backslash B^{0}(S)\right) \backslash C(A)=\varnothing$ and the equality $\pi x=B(x) \backslash B^{0}(S)$ is established. By Lemma 2, $\pi x \subset F(D)$ and (a) is proved.

From (a) we deduce $\mathrm{Cl} \pi S \subset F(D)$, since the last set is closed. For the converse, if $y \in F(D)$, then $y \in \mathrm{Cl} D$ and so there exists a sequence $\left\{x_{n}\right\}$ with $x_{n} \in D$ and $\left\{x_{n}\right\} \rightarrow y$. Let $y_{n} \in \pi x_{n}$; then $d\left(y, y_{n}\right) \leq d\left(y, x_{n}\right)$ 
$+d\left(x_{n}, y_{n}\right)$. Since $\left\{x_{n}\right\} \rightarrow y, r\left(x_{n}\right) \rightarrow 0$ and hence also $d\left(y, y_{n}\right) \rightarrow 0$. By Lemma 3, $y_{n} \in \pi S$ and thus $y \in \mathrm{Cl} \pi S$.

Lemma 6. If $D$ is the convex deficiency of $A$, then $D \subset C(F(D))$ and $C(D)=C(F(D))$.

Proof. Suppose $x \in D$ but $x \notin C(F(D))$. Project $x$ onto $z \in C(F(D))$ and let $H_{z}$ be the supporting hyperplane of $C(F(D))$ at $z$ orthogonal to $[z, x]$ and let $E_{z}$ be the corresponding closed half space containing $C(F(D))$. We claim that $C(A) \subset E_{z}$. If not, then there exists some $y \in A \backslash E_{z}$. Because $x \in C(A),[x, y]$ lies in $C(A)$. Now $[x, y] \cap A$ is closed and nonempty and we let $y^{\prime}$ be the unique point of $[x, y] \cap A$ nearest $x$. Then $\left[x, y^{\prime}\right) \subset D$ and $y^{\prime} \in F(D)$, contradicting the inclusions $F(D) \subset C(F(D)) \subset E_{z}$. Hence all points of $A$ and of $C(A)$ lie in $E_{z}$, in particular, $x \in D \subset C(A)$, contradicting our assumption. Hence $D \subset C(F(D))$. But then $C(D) \subset C(F(D)) \subset C(\mathrm{Cl} D)=C(D)$ and the second statement follows.

We can now terminate the proof of the theorem. Assume that $A$ and $A^{\prime}$ have same skeletal pair $(S, q)$. Then, by Lemma $5(\mathrm{a}), \pi x=\pi^{\prime} x$ for each $x \in S$ and consequently $P(A)=P\left(A^{\prime}\right)$. Thus by Lemmas 3 and 5(b), $D^{*}=D^{* *}, F(D)=F\left(D^{\prime}\right)$. But by Lemma 6 then $C(D)$ $=C(F(D))=C\left(D^{\prime}\right)$ and $D=C(D) \cap D^{*}=D^{\prime}$.

\section{REFERENCES}

1. G. Bouligand, Introduction a la gémétrie infinitésmale directe, Vuibert, Paris, 1932.

2. L. Calabi and W. E. Hartnett, Shape recognition, prairie fires, convex deficiencies and skeletons, Amer. Math. Monthly 75 (1968), 335-342.

3. T. Motzkin, Sur quelques propriêtés charactéristiques des ensembles convexes, Atti Accad. Naz. Lincei Rend. Cl. Sci. Fis. Mat. Natur. 21 (1935), 562-567.

4. - Sur quelques propriétés charactéristiques des ensembles barnés non convexes, Atti Accad. Naz. Lincei Rend. Cl. Sci. Fis. Mat. Natur. 21 (1935), 773-779.

5. F. A. Valentine, Convex sets, McGraw-Hill, New York, 1964.

Parke Mathematical Laboratories, Inc. 